\title{
Twenty years of paediatric intestinal failure-associated liver disease - experience of a transplant centre
}

\author{
V. Iyer ${ }^{1}$, S. V. Beath ${ }^{1}$, H. Gowen ${ }^{2}$ and G. Gupte ${ }^{1}$ \\ ${ }^{1}$ The Liver Unit, Birmingham Children's Hospital and ${ }^{2}$ British Intestinal Failure Registry, Institute of Child Health
}

Over 1000 children annually receive parenteral nutrition (PN) for at least 4 weeks and about 70 go on to receive home PN each year ${ }^{(1)}$. Most of these children have biochemical abnormalities of liver function at some point during the course of their treatment although only a minority develop life-threatening intestinal failure associated liver disease (IFALD). The onset of IFALD is an indication for referral for consideration of small-bowel transplantation (SBTx). The decision to recommend SBTx depends on many factors, but one of the key issues is estimating the severity of IFALD and the possibility of clinical deterioration.

We reviewed our database containing clinical information on all children undergoing a formal transplant assessment from 1990 until 2009 to ascertain the number with IFALD. Bilirubin concentrations recorded at the time of SBTx assessment have been used to assign a severity category for IFALD based on the criteria from Xth International Symposium of Small Bowel transplantation ${ }^{(2)}$.

\begin{tabular}{|c|c|c|c|c|}
\hline Bilirubin and IFALD category & $1990-1994$ & 1995-1999 & $2000-2004$ & $2005-2009$ \\
\hline Bilirubin $<50 \mu \mathrm{mol} / 1-$ no IFALD and Type 1 & $7(32 \%)$ & $14(29 \%)$ & $36(23 \%)$ & $20(23 \%)$ \\
\hline Bilirubin $50-100 \mu \mathrm{mol} / \mathrm{l}$ - Type 2 IFALD & $2(9 \%)$ & $4(8 \%)$ & $1(3 \%)$ & $3(3 \%)$ \\
\hline Bilirubin $101-200 \mu \mathrm{mol} / \mathrm{l}$ - Type 3 IFALD & $2(9 \%)$ & $6(12 \%)$ & $15(16 \%)$ & $21(24 \%)$ \\
\hline Bilirubin $>200 \mu \mathrm{mol} / 1-$ Type $3+$ IFALD & $10(45 \%)$ & $24(49 \%)$ & $40(43 \%)$ & $44(50 \%)$ \\
\hline Total & 21 & 48 & 92 & 88 \\
\hline
\end{tabular}

Overall 123 children were considered stable and were not offered SBTx, 37 subsequently died of causes other than IFALD. Of the 77 children ( $31 \%$ of those referred) with no IFALD or Type 1 IFALD, 13 were transplanted (for loss of access for feeding catheters). Of the 118 children with type $3+$ IFALD, 32 died on the transplant waiting list, 42 received a transplant. Thirty children were not offered SBTx because of contra-indications and 28 of those children died. Fifty-four children (21\%) had less severe IFALD (types 2 and 3 ) at the time of referral and the outcome for them was: 7 died on transplant waiting list; 21 received a transplant. 16 showed an improvement in IFALD and were not put on the waiting list for transplant. The 5-year survival for SBTx in patients with no IFALD or type 1 IFALD, is now $80 \%$. However, the largest group of referred patients had type $3+$ IFALD and despite the increased morbidity and mortality of SBTx in the context of liver failure, 118 children $(47 \%)$ were already in that condition at the time of referral. These data suggest that type 2 IFALD can progress rapidly to types 3 and $3+$ IFALD and that patients would benefit from earlier referral to a transplant centre.

1. Köglmeier J, Day C \& Puntis JWL (2008) Clinical outcome in patients from a single region who were dependent on parenteral nutrition for 28 days or more. Arch Dis Child 93, 300-302.

2. Beath S, Pironi L, Gabe S et al. (2008) Collaborative strategies to reduce mortality and morbidity in patients with chronic intestinal failure including those who are referred for small bowel transplantation. Transplantation 85, 1378-1384. 